\title{
Improved Performance of the Piezoelectric Monomorph with Perpendicular Electrode Connections for Sensing and Energy Harvesting
}

\author{
Ming Ma, Zhenrong Li, Xiaoyong Wei, Zhuo Xu, and Xi Yao \\ Electronic Materials Research Laboratory, Key Laboratory of the Ministry of Education \& International Center for Dielectric Research, \\ Xian Jiaotong University, Xian 710049, China
}

Correspondence should be addressed to Zhenrong Li; zhrli@mail.xjtu.edu.cn

Received 18 January 2013; Accepted 18 March 2013

Academic Editor: Xiaoning Jiang

Copyright (C) 2013 Ming Ma et al. This is an open access article distributed under the Creative Commons Attribution License, which permits unrestricted use, distribution, and reproduction in any medium, provided the original work is properly cited.

\begin{abstract}
Piezoelectric monomorph, which has only one element, is a potential structure for piezoelectric applications in some extreme conditions. But as the restriction of the strain neutral layer, the traditional parallel electrode connection is not effective for sensing and energy harvesting. In this paper, perpendicular electrode connections were designed to utilize the nonuniform shear piezoelectric effect in the cross section of the monomorph, which made the monomorph avoid the restriction of the strain neutral layer. The PZT5 ceramic monomorph was preliminarily studied in this experiment. By comparing seven forms of perpendicular electrode connections with the traditional parallel electrode connection, the whole superposed perpendicular electrode connection is considered as the optimal output way for the monomorph. It can produce $13 \mathrm{~V}$ peak-to-peak (pk-pk) voltage in open circuit and $14.56 \mu \mathrm{W}$ maximum power with the matching resistance, which are much more than the parallel electrode connection $0.78 \mathrm{~V}$ and $0.14 \mu \mathrm{W}$.
\end{abstract}

\section{Introduction}

Piezoelectric cantilever is widely used in the piezoelectric device for sensing and energy harvesting [1-6]. At room temperature and ambient pressure, unimorph and bimorph, which utilize the lateral extensional piezoelectric effect, are considered as a promising solution for sensing and energy harvesting $[7,8]$. But when both of the above composite structures are used under the relative tough environment, such as high temperature and high pressure or low temperature and low pressure, the stability and performance of the device will be lower as the thermal-oxidative degradation and the low temperature brittleness of the bonding materials between different components. Even in the normal situation, the internal stresses and the permanent strain in the bonding layer will lead to the rapid deterioration of the device after long time using [9].

As for the simplest transducer that has only one piezoelectric component, the piezoelectric monomorph avoids the bonding issues and is considered as the only solution in the extreme conditions. But the existence of the strain neutral layer, which is in the middle of the thickness of the piezoelectric element, neutralizes the positive and negative induced charges [10]. As a result, with the traditional parallel electrode connection, the monomorph is not effective in the piezoelectric applications. In order to improve the performance of the monomorph, many studies have been focused on the nonuniform polarization inside the piezoelectric materials. Based on the nonuniform distribution of the electric field caused by the semiconductor-metal electrode contact, a monomorph of semiconductive piezoelectric ceramics was researched as an actuator, which can make a bend curvature comparable to bimorph [11]. With a ferroelectric inversion layer caused by heat treatment at a lower temperatures than the Curie point, a $\mathrm{LiNbO}_{3}$ monomorph was studied to make the piezoelectric device without the bonding layer [12]. Monomorph with functional gradients materials was researched because of the constitutional gradation between the composite components, which made the strain neutral layer deviate from the middle of the thickness [13]. Recently, 


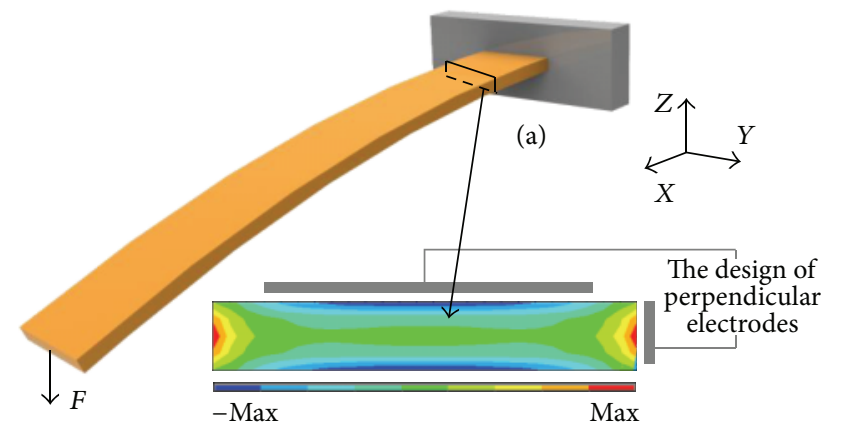

(b)

Figure 1: (a) The bending piezoelectric monomorph, and (b) the nonuniform shear strain distribution in the cross section (color online).

the piezoelectric monomorph with interdigitated electrodes was studied, which utilized the nonuniform $d_{33}$ coupling along the length to make the monomorph more effective in piezoelectric application $[14,15]$.

In this work, the piezoelectric monomorph with perpendicular electrode connections was researched preliminarily to utilize the nonuniform shear piezoelectric effect in the cross section for sensing and energy harvesting.

As shown in Figure 1, when the cantilever is bent by a tip force $F$, there is a nonuniform distribution of the shear strain $S_{x z}$ along the width, which is the result of the uneven stresses near the fixed end. Meanwhile, the maximum shear strains concentrate near the four surfaces of the monomorph and are opposite between the horizontal surface and the vertical surface. In order to utilize the nonuniform shear strain, the piezoelectric monomorph with perpendicular electrodes is designed. The nonuniform shear piezoelectric effect can be presented as

$$
D_{p}=e_{p x z} S_{x z}
$$

where $D_{p}$ is the electric displacement that is utilized by the perpendicular electrodes, $S_{x z}$ is the nonuniform shear strain in the cross section of the bending monomorph, and $e_{p x z}$ is the piezoelectric constant that presents the nonuniform piezoelectric effect between the perpendicular electrodes.

\section{Experimental Procedure}

In the experiment, a PZT ceramic sheet with the length of $L=35 \mathrm{~mm}$, thickness of $T=0.8 \mathrm{~mm}$, and width of $W=$ $5 \mathrm{~mm}$ was used as a prototype (Figure 2(a)). The sheet was polarized along the thickness direction with the electric field of $3 \mathrm{kV} / \mathrm{mm}$ at $100^{\circ} \mathrm{C}$, and the piezoelectric coefficient $\mathrm{d}_{31}$ is $-187 \times 10^{-12} \mathrm{C} / \mathrm{N}$. Then, the top and bottom faces of the sheet, which were perpendicular to the $z$-axis, were painted with the partial room temperature silver electrodes that were $W_{2}=3 \mathrm{~mm}$ wide and spaced $W_{1}=W_{3}=1 \mathrm{~mm}$ width with two edges, respectively. The whole covered room temperature silver electrodes were painted on both of the side faces that were perpendicular to the $y$-axis. Four wires were leaded from each electrode of the sheet. The identifier of each wire was shown in Figure 2(b).

The experimental setup is shown in Figure 3(a). The mechanical shaker (Nanjing Foneng Co., HEV200), which was used to vibrate the monomorph, was driven by an arbitrary function generator (NF WF1974) and a power amplifier (NF HSA4014). The output voltage of the monomorph was monitored by an oscilloscope (Tektronix TDS2022B). The acceleration of the base was measured by a piezoelectnic accelerometer and a charge amplifier. Then the monomorph was clamped on the base with a tip mass as a cantilever. In Figure 3(b), there are eight forms of electrode connections. Connection 1-2 was the conventional parallel electrode connection, which was used to be compared with perpendicular electrode connections. As a result of the symmetry of the structure, there were four perpendicular electrode connections in the monomorph, which include connection 1-3, connection 1-4, connection 2-3, and connection 2-4. Based on the above analysis that both side faces of the monomorph had the same charge distribution, the partial superposed perpendicular electrode connections were made by superposing both side wires, which include connection 1-34 and connection 2-34. Then by superposing the top and bottom wires of the partial superposed perpendicular electrode connections, the whole superposed perpendicular electrode connection (connection 12-34) was made.

\section{Results and Discussion}

Based on the above eight forms of electrode connections, the peak-to-peak (pk-pk) open circuit voltages of the PZT ceramic monomorph without tip mass, with $0.7 \mathrm{~g}$, and $1.4 \mathrm{~g}$ tip mass were tested at the resonance frequencies $414 \mathrm{~Hz}$, $227 \mathrm{~Hz}$, and $171 \mathrm{~Hz}$, respectively. The vibration acceleration was $11 \mathrm{~m} / \mathrm{s}^{2}$. As shown in Figure 4, more tip masses made the monomorph with increased prestress and lower resonant frequency, which induced higher voltage. However, even with $1.4 \mathrm{~g}$ tip mass, the monomorph with connection 1-2 only produced less than $1 \mathrm{~V}$ pk-pk voltage in open circuits, which implied that the parallel electrode connection is not effective for piezoelectric output. In comparison, the perpendicular electrode connections can produce $9.37 \mathrm{~V} \mathrm{pk-pk}$ open circuit voltages with $1.4 \mathrm{~g}$ tip mass, which is more effective than connection 1-2. The output voltages of four perpendicular electrode connections that were in the same level also corroborated that both side faces of the monomorph had the same charge distribution. By superposing the induced charge of two side faces, the partial superposed perpendicular electrode connections included connection 1-34 and connection 2-34. They can produce $12.6 \mathrm{~V}$ pk-pk voltage with $1.4 \mathrm{~g}$ tip mass. This was because the superposition of the perpendicular electrode connections increased the effective area of the charge distribution, which made the monomorph produce higher voltage. In order to make full use of the induced charges, the whole superposed perpendicular electrode connection (connection 12-34) was made. It can produce $13 \mathrm{~V}$ pk-pk voltage with $1.4 \mathrm{~g}$ tip mass, which 


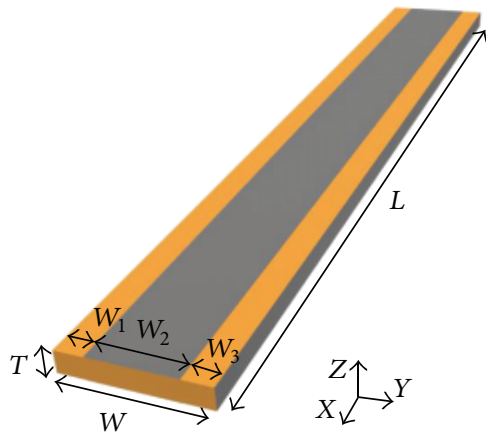

Piezoelectric material Silver electrodes

(a)

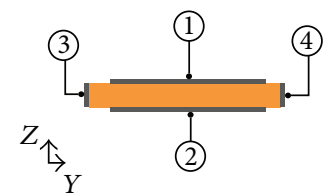

Piezoelectric material Silver electrodes

(b)

Figure 2: (a) Dimensions of the prototype monomorph with perpendicular electrodes, and (b) four wires are leaded from each electrode. Identifier of each leaded wire: no. 1 and no. 2 wires are leaded from the top and bottom electrode respectively, no. 3 wire is leaded from one of the side electrodes, and no. 4 wire is leaded from the other one (color online).
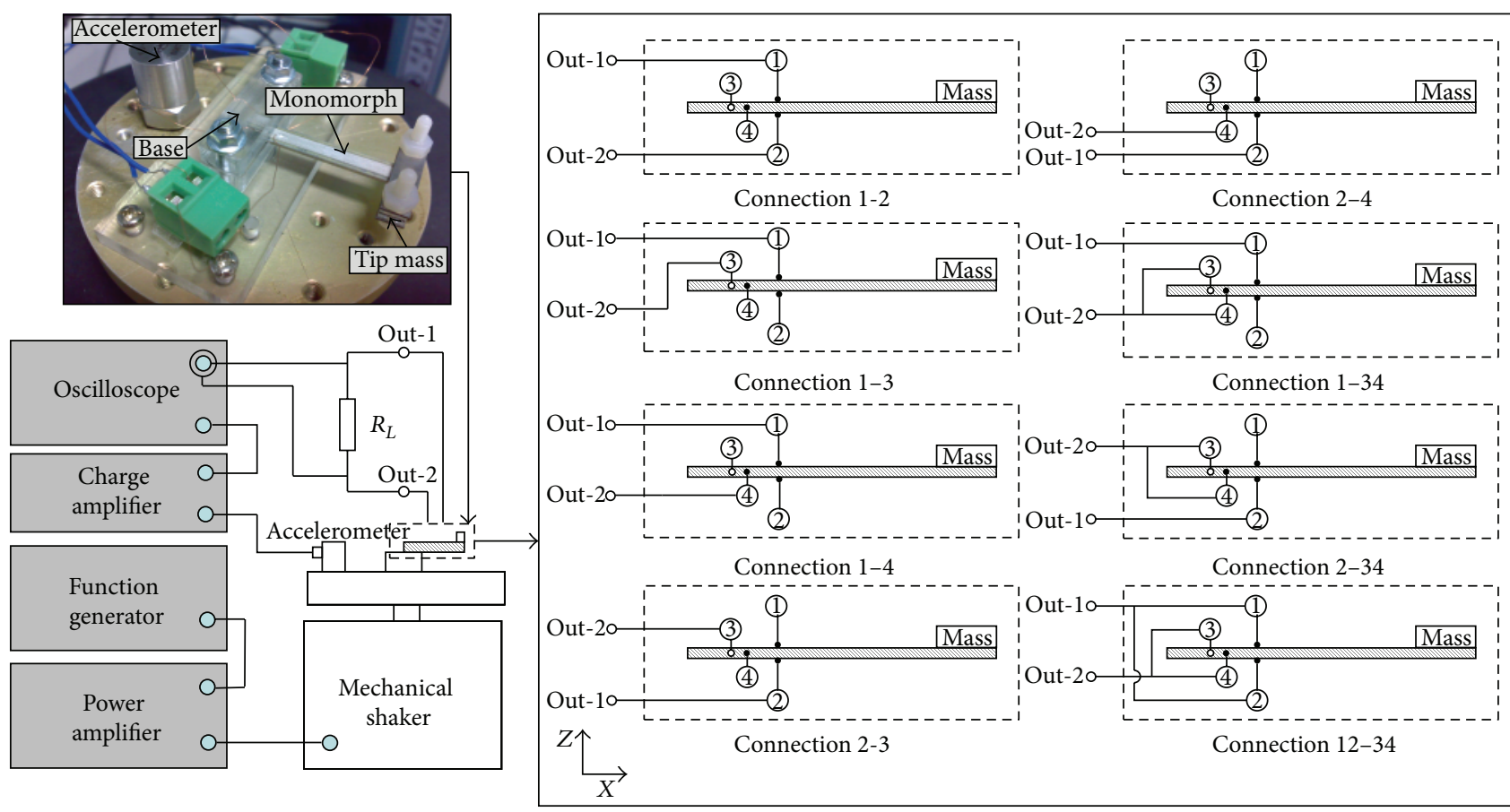

(a)

- Back electrode

- Front electrode

Figure 3: (a) The experimental setup and the clamped monomorph. (b) Eight forms of electrode connections. The parallel electrode connection: connection 1-2. Four perpendicular electrode connections: connection 1-3, 1-4, 2-3, and 2-4. Two partial superposed perpendicular electrode connections: connection 1-34 and 2-34. The whole superposed perpendicular electrode connection: connection 1234. Connection 1-2 means that no. 1 wire is connected to the out-1 test point, and no. 2 wire is connected to the out- 2 test point. Connection 1-3 (similar to connection 1-4, 2-3, and 2-4) means that no. 1 wire is connected to the out-1 test point, and no. 3 wire is connected the out-2 test point. Connection 1-34 (similar to connection 2-34) means that no. 1 wire is connected to the out-1 test point, and no. 3 wire and no. 4 wire are connected to the out- 2 test point. Connection 12-34 means that no. 1 and no. 2 wires are connected to the out-1 test point, and no. 3 no. 4 wires are connected to the out-2 test point (color online). 


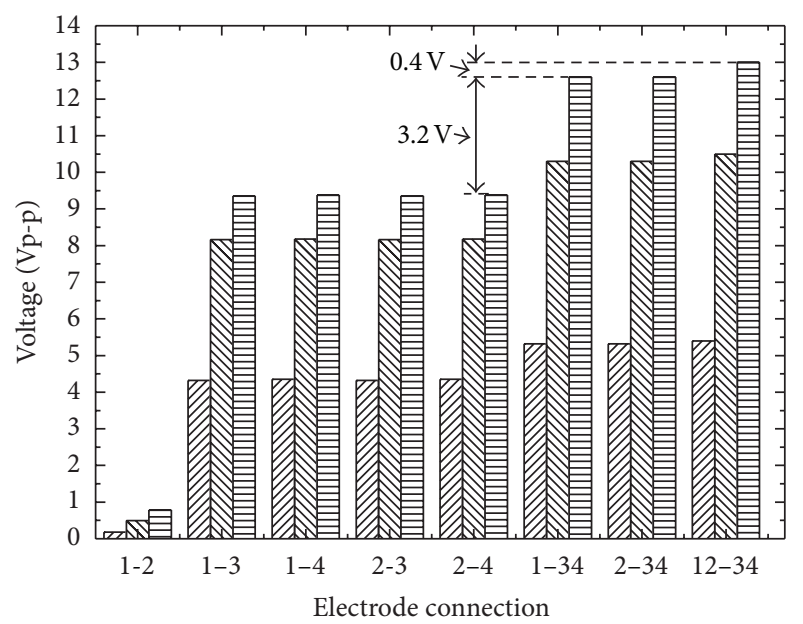

שIIA Without tip mass

With $0.7 \mathrm{~g}$ mass

E With $1.4 \mathrm{~g}$ mass

FIGURE 4: The pk-pk voltage in open circuit of various electrode connections with different tip masses, when the piezoelectric monomorph was vibrated under an acceleration of $11 \mathrm{~m} / \mathrm{s}^{2}$ at the respective resonance frequencies.

was considered as the optimal piezoelectric output way of the monomorph with perpendicular electrodes. In addition, when the top and bottom electrodes of the monomorph were superposed, there was only $0.4 \mathrm{~V}$ pk-pk voltage increased from connection 12-34 to connection 1-34. By contrast, when both side electrodes were superposed, there was $3.2 \mathrm{~V} \mathrm{pk-}$ pk voltage increased from connection 1-34 to connection 13. This indicates that the side electrodes make the primary contribution for the output voltage of the perpendicular electrode connection.

The pk-pk voltages and output power of various connections under external load resistances were tested with $11 \mathrm{~m} / \mathrm{s}^{2}$ acceleration and $1.4 \mathrm{~g}$ tip mass at $171 \mathrm{~Hz}$. As shown in Figure 5(a), the pk-pk voltages of various electrode connections increase as the external resistance increases and then tend to be stable. Connection 1-2 produced the pk-pk voltage in lower level than others, which tended to be stable after $300 \mathrm{k} \Omega$. For connection 1-3, the pk-pk voltage tended to be stable after $3 \mathrm{M} \Omega$. It indicates that the internal impedance of the perpendicular electrode connection is larger than the parallel electrode connection. However, as specified in Figure 5(a), even with the resistance less than $100 \mathrm{k} \Omega$, the perpendicular electrode connection also can produce a higher voltage than the parallel electrode connection.

The output power is calculated by the formula $P=$ $U_{\text {pk-pk }}^{2} / 8 R_{L}$, where $U_{\text {pk-pk }}$ is the pk-pk voltage, and $R_{L}$ is the external load resistance. As shown in Figure 5(b), the power increases with the increasing external resistance, and reaches the maximum value with the matching resistance, then decreases. Compared with connection 1-2, which produced the maximum power of only $0.14 \mu \mathrm{W}$ with the matching resistance about $100 \mathrm{k} \Omega$, the maximum output power of connection $1-3$ can be $4.41 \mu \mathrm{W}$ with $800 \mathrm{k} \Omega$. As specified in

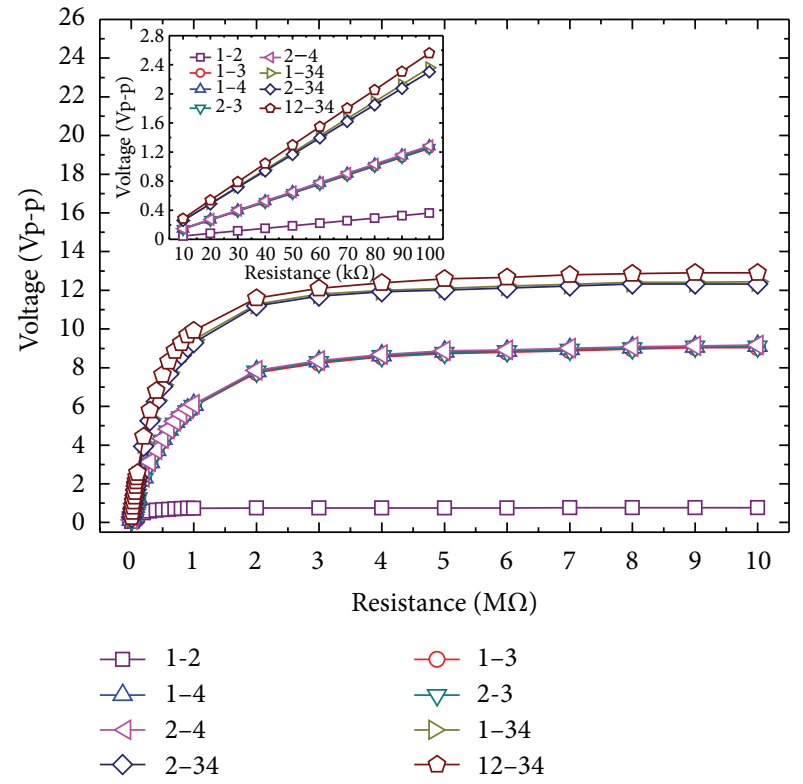

(a)

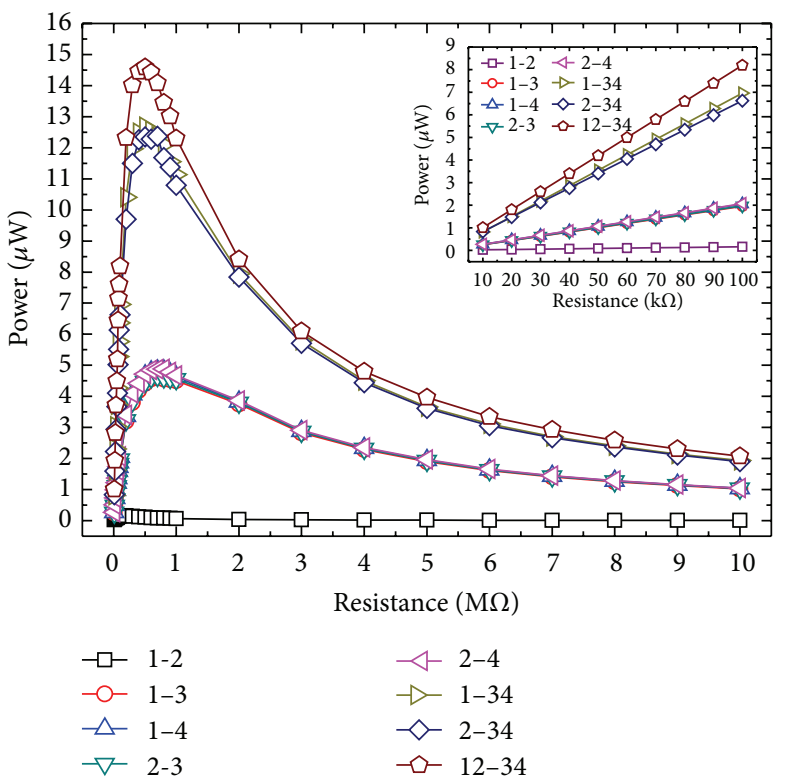

(b)

FIGURE 5: (a) The pk-pk voltage and (b) the output power of eight electrode connections under various external resistances, when the piezoelectric monomorph was vibrated under an acceleration of $11 \mathrm{~m} / \mathrm{s}^{2}$ at $171 \mathrm{~Hz}$ with $1.4 \mathrm{~g}$ tip mass.

Figure 5(b), even with a lower external load resistance that is less than $100 \mathrm{k} \Omega$, the output power of the perpendicular electrode connection is also more than the traditional parallel electrode connection. This indicates that the perpendicular electrode connection is more effective than the conventional parallel electrode connection for energy harvesting, which is the result of avoiding the restriction of the stain neutral layer. Regarding the superposition of the perpendicular electrode connection, connection 12-34 can produce the maximum 
power $14.56 \mu \mathrm{W}$ with the matching resistance, which is a little more than connection $1-34$ that produces $12.67 \mu \mathrm{W}$. The whole superposed perpendicular electrode connection can maximize the piezoelectric output of the piezoelectric monomorph to harvest the vibration energy. Moreover, by comparing the maximum power between connection 1234 and connection $1-34$, there was only $1.4 \mu \mathrm{W}$ increased. It indicates that the superposition of the top and bottom electrodes also has less effect on the output power of the monomorph than that of the side electrodes.

\section{Conclusions}

In conclusion, the nonuniform shear piezoelectric effect, which was utilized by the perpendicular electrode connections, was studied in the PZT5 ceramic monomorph. The output voltage and power can be improved by perpendicular electrode connections resulting from avoiding the restriction of the strain neutral layer. By comparing the output of eight forms of electrode connection, the whole superposed electrode connection was considered as the optimal way of the monomorph for higher voltage and more power. In the specific application environments, which require avoiding the bonding issues or the restriction of the strain neutral layer, the monomorph with perpendicular electrode connections can be considered as a solution. In addition, in order to maximize the efficiency of the energy converting, further work on the strain distribution of the nonuniform shear piezoelectric effect in the monomorph is required.

\section{Acknowledgments}

This work was financially supported by the National Basic Research Program of China (973 program) (Grant no. 2009CB623306) and the International Science and Technology Cooperation Program of China (Grant no. 2010DFR 50480).

\section{References}

[1] H. A. Sodano, G. Park, and D. J. Inman, "Estimation of electric charge output for piezoelectric energy harvesting," Strain, vol. 40, no. 2, pp. 49-58, 2004.

[2] S. Roundy, E. S. Leland, J. Baker et al., "Improving power output for vibration-based energy scavengers," IEEE Pervasive Computing, vol. 4, no. 1, pp. 28-36, 2005.

[3] S. Roundy, "On the effectiveness of vibration-based energy harvesting," Journal of Intelligent Material Systems and Structures, vol. 16, no. 10, pp. 809-823, 2005.

[4] S. Priya, "Advances in energy harvesting using low profile piezoelectric transducers," Journal of Electroceramics, vol. 19, no. 1, pp. 165-182, 2007.

[5] Y. C. Shu and I. C. Lien, "Analysis of power output for piezoelectric energy harvesting systems," Smart Materials and Structures, vol. 15, no. 6, article 001, pp. 1499-1512, 2006.

[6] Y. C. Shu and I. C. Lien, "Efficiency of energy conversion for a piezoelectric power harvesting system," Journal of Micromechanics and Microengineering, vol. 16, no. 11, pp. 2429-2438, 2006.
[7] J. W. Xu, W. W. Shao, F. R. Kong, and Z. H. Feng, "Rightangle piezoelectric cantilever with improved energy harvesting efficiency," Applied Physics Letters, vol. 96, no. 15, Article ID 152904, 2010.

[8] F. Goldschmidtboeing and P. Woias, "Characterization of different beam shapes for piezoelectric energy harvesting," Journal of Micromechanics and Microengineering, vol. 18, no. 10, Article ID 104013, 2008.

[9] K. Uchino, "Materials issues in design and performance of piezoelectric actuators: an overview," Acta Materialia, vol. 46, no. 11, pp. 3745-3753, 1998.

[10] X. Gao, W. H. Shih, and W. Y. Shih, "Induced voltage of piezoelectric unimorph cantilevers of different nonpiezoelectric/piezoelectric length ratios," Smart Materials and Structures, vol. 18, no. 12, Article ID 125018, 2009.

[11] K. Uchino, "Monomorph characteristics in lead zirconate based ceramics," Japanese Journal of Applied Physics, vol. 26, pp. 201203, 1987.

[12] K. Nakamuram, H. Ando, and H. Shimizu, "Bending vibrator consisting of a $\mathrm{LiNbO} 3$ plate with a ferroelectric inversion layer," Japanese Journal of Applied Physics, vol. 26, pp. 198-200, 1987.

[13] X. Zhu, J. Zhu, S. Zhou et al., "Actuators, piezoelectric ceramics and functionally gradient materials," Ferroelectric, vol. 263, pp. 67-76, 2001.

[14] Y. K. Hong, H. K. Park, S. Q. Lee, K. S. Moon, R. R. Vanga, and M. Levy, "Design and performance of a self-sensing, selfactuating piezoelectric monomorph with interdigitated electrodes," in Optomechatronic Sensors, Actuators, and Control, vol. 6048 of Proceedings of SPIE, pp. 210-217, October 2004.

[15] Y. K. Hong and K. Moon, "Single crystal piezoelectric transducers to harvest vibration energy," in Optomechatronic Actuators and Manipulation, vol. 6048 of Proceedings of SPIE, no. 60480E, December 2005 

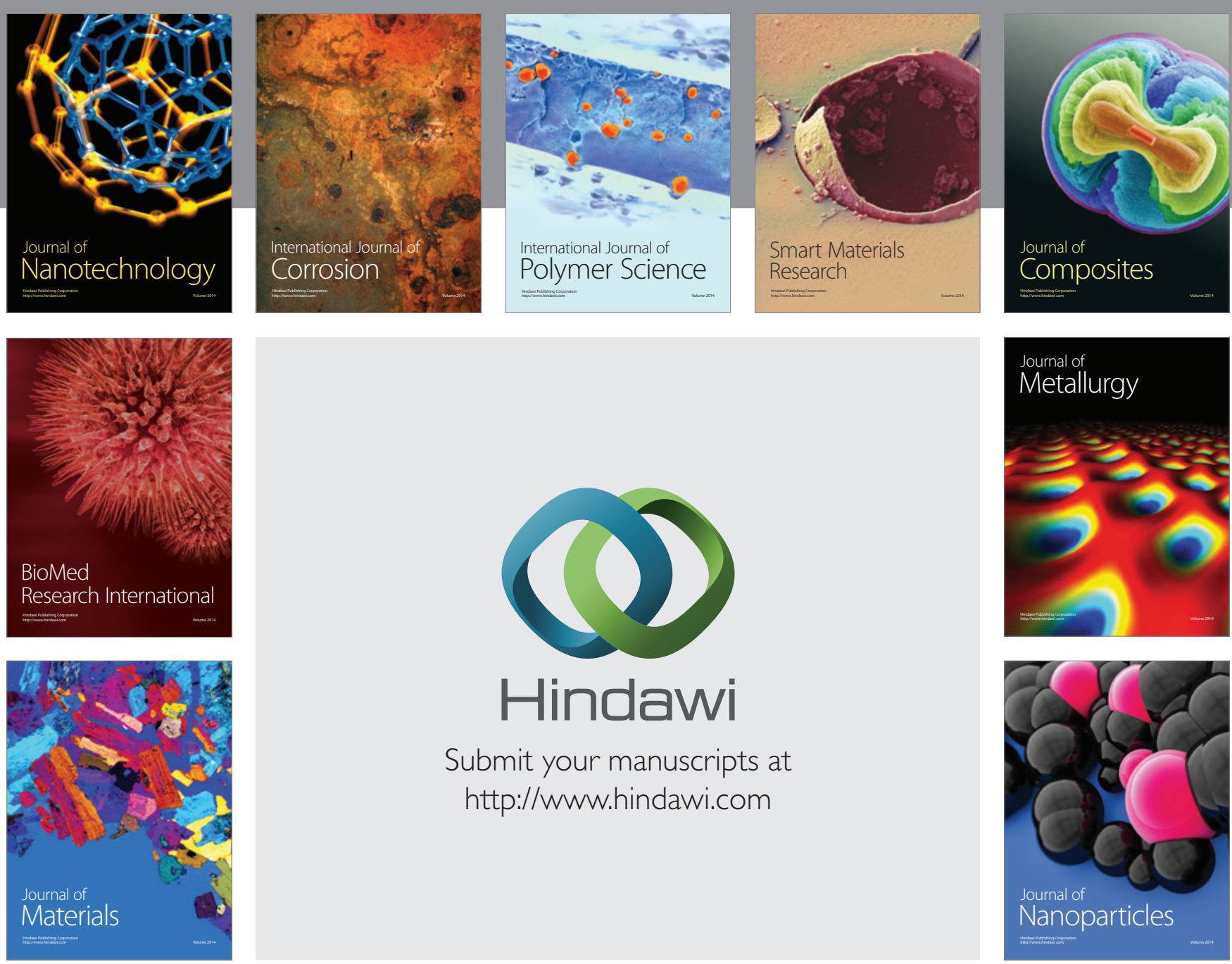

Submit your manuscripts at http://www.hindawi.com
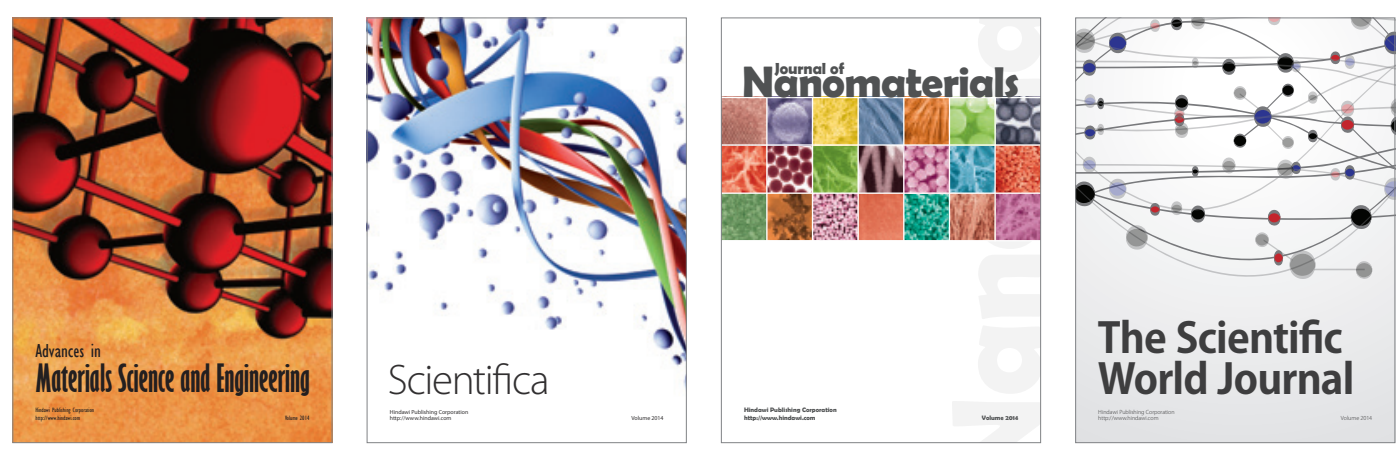

\section{The Scientific World Journal}
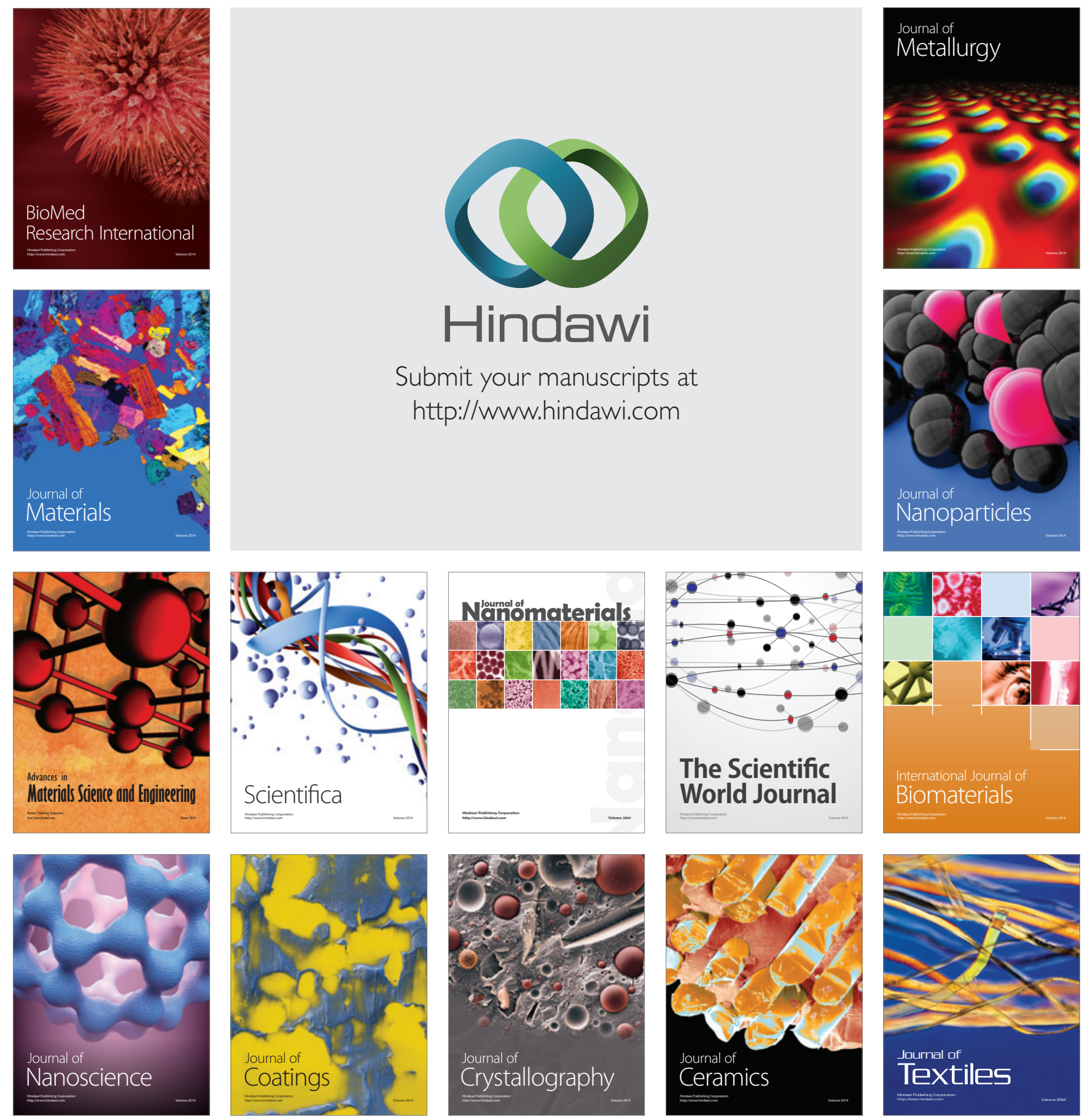